\title{
Anwendbarkeit des Arbeitsgesetzes (ArG) auf Spitäler und Spitalärzte
}

\author{
U. Reinhard
}

Korrespondenz:

lic. iur. Urs Reinhard Rechtsberatungsstelle Verein der Leitenden Spitalärzte der Schweiz VLSS Gutenbergstrasse 9 CH-3011 Bern
Auf den 1. Januar 2005 haben das Arbeitsgesetz und einige seiner Verordnungen Änderungen erfahren, die auf die Anstellungsverhältnisse von Spitalärztinnen und Spitalärzten nicht ohne Einfluss bleiben werden. Das Arbeitsgesetz zeichnet sich durch die Festlegung einer Vielzahl von Grundsätzen, Ausnahmen von diesen Grundsätzen und schliesslich Ausnahmen von den Ausnahmen aus, was seine Leserlichkeit nicht eben erleichtert. Der vorliegende Artikel soll helfen, die Rechtslage zu klären und anhand einer Tabelle verständlich darzustellen, wie die einzelnen Spitalärztinnen und Spitalärzte vom Arbeitsgesetz erfasst werden.

Bitte wenden Sie sich auch bei weiteren allgemeinen Rechtsfragen an die Rechtsauskunftsstelle des VLSS. Bei Fragen zur 2. Säule gibt Ihnen ausserdem Frau Lotti Gautschi bei der Pendia Associates AG, Tödistrasse 38, Postfach 2056, 8027 Zürich, Tel. 04428920 06, E-Mail: lotti. gautschi@pendia.ch, gerne Auskunft.

\section{Anwendbarkeit des Arbeitsgesetzes auf einzelne Betriebe}

Art. 1 Abs. 1 des ArG hält fest, dass grundsätzlich sämtliche öffentlichen und privaten Betriebe dem Gesetz unterstellt werden. Von diesem Grundsatz gibt es Ausnahmen. Gemäss Art. 2 Abs. 1 ArG gilt das ArG u.a. nicht für die Verwaltungen des Bundes, der Kantone und der Gemeinden (lit. a). Damit ist die Zentralverwaltung gemeint, welche hierarchisch gegliedert und fast ausschliesslich dem betreffenden öffentlichen Recht unterworfen ist.

Vorbehalten bleibt Abs. 2 von Art. 2 ArG. Er besagt, dass mittels einer Verordnung festgelegt werden soll, welche öffentlichen Anstalten den Verwaltungen des Bundes, der Kantone und der Gemeinden gleichzustellen seien und damit wie diese vom ArG nicht erfasst werden sollen, und welche Betriebe des Bundes, der Kantone und der Gemeinden entgegen Art. 1 Abs. 1 ArG ausnahmsweise doch vom Gesetz erfasst werden sollen. Dies geschieht durch die Verordnung 1 zum Arbeitsgesetz [ArGV 1].
Art. 7 dieser Verordnung legt fest, dass die öffentlich-rechtlichen Anstalten ohne Rechtspersönlichkeit (z.B. Kantonsspitäler) dem ArG grundsätzlich nicht unterstellt sind.

Es bestehen zwei Ausnahmen zu diesem Grundsatz (sogenannte «Ausnahmen der Ausnahmen»), wo auch solche öffentlich-rechtlichen Anstalten ohne Rechtspersönlichkeit vom ArG erfasst werden:

a) Ist die Mehrheit der Arbeitnehmer einer öffentlich-rechtlichen Anstalt ohne Rechtspersönlichkeit auf der Grundlage eines privatrechtlichen Einzelarbeitsvertrages angestellt, so gilt das ArG für den gesamten Betrieb (insbesondere auch die Vorschriften über Arbeits- und Ruhezeit) (Abs. 1).

b) Das ArG gilt auch für die privatrechtlich angestellte Minderheit der Arbeitnehmer einer öffentlich-rechtlichen Anstalt ohne Rechtspersönlichkeit mit überwiegend öffentlichrechtlich angestellten Arbeitnehmern. Das ArG gilt für diese Minderheit hingegen dann nicht, wenn das öffentliche Dienstrecht für den betroffenen Arbeitnehmer günstigere Bedingungen vorsieht (Abs. 2).

Seit Anfang Jahr besteht zudem eine weitere wichtige Ausnahme: Der im Zuge einer Gesetzesänderung auf den 1. Januar 2005 neu eingefügte Art. 4a der ArGV 1 besagt, dass das ArG für öffentlich-rechtliche Spitäler ohne Rechtspersönlichkeit gilt, wenn es um die Arbeitsverhältnisse von Assistenzärztinnen und -ärzten geht.

In jedem Fall und immer anwendbar bleiben gemäss Art. 3a ArG die Vorschriften über den Gesundheitsschutz (Art. 6, 35 und 36a) (Anpassung ans EU-Recht).

Aus der Grundregel in Art. 1 Abs. 1 ArG und dem Umkehrschluss aus Art. 7 Abs. 1 ArGV 1 folgt, dass öffentlich-rechtliche Anstalten mit eigener Rechtspersönlichkeit (z. B. das Universitätskinderspital beider Basel UKBB) dem ArG integral unterstellt sind. Sie sind aus der Zentralverwaltung ausgegliedert, deren öffentlich-rechtlicher Organisation entzogen und organisieren sich häufig privatwirtschaftlich, womit sie keinen dem ArG gleichwertigen Schutz zu gewährleisten vermögen. 


\section{Vorbehalt des öffentlichen Dienstrechts}

Art. 71 lit. b ArG enthält einen allgemeinen Vorbehalt der Vorschriften des Bundes, der Kantone und der Gemeinden über das öffentlich-rechtliche Dienstverhältnis. Dieser Vorbehalt besagt, dass primär das massgebliche eidgenössische, kantonale oder kommunale Recht gilt. Erst wenn dieses zu einer konkreten Frage keine Regelung enthält, gelangt das ArG zur Anwendung.

Auch hier besteht eine wichtige Ausnahme: Die Vorschriften des ArG über den Gesundheitsschutz und neu seit 1 . Januar 2005 auch diejenigen über die Arbeits- und Ruhezeit gelten als Mindeststandard. Die im eidgenössischen, kantonalen oder kommunalen Recht vorgesehene Regelung findet also nur dann Anwendung, wenn sie für den Arbeitnehmer besser ist als diejenige im ArG.

\section{Anwendbarkeit des Arbeitsgesetzes auf einzelne Ärztinnen und Ärzte an den Spitälern}

Für alle vom Anwendungsbereich des ArG betroffenen Spitäler muss Art. 3 ArG beachtet werden, der gewisse Arbeitnehmer vom Geltungsbereich ausnimmt:

a) Gemäss Art. 3 lit. d sind ausgenommen Arbeitnehmer, die eine höhere leitende Tätigkeit oder eine rein wissenschaftliche Tätigkeit ausüben. In Anlehnung an Art. 9 ArGV 1 folgt daraus, dass Chefärzte und auch Leitende Ärzte vom ArG prinzipiell nicht erfasst werden. Allerdings muss hier genauer präzisiert werden:

Der Begriff der höheren leitenden Tätigkeit wird durch Art. 9 ArGV 1 konkretisiert. Danach übt eine höhere leitende Tätigkeit aus, «wer auf Grund seiner Stellung und Verantwortung sowie in Abhängigkeit von der Grösse des Betriebes über weitreichende Entscheidungsbefugnisse verfügt oder Entscheide von grosser Tragweite massgeblich beeinflussen und dadurch auf die Struktur, den Geschäftsgang und die Entwicklung eines Betriebes oder Betriebsteils einen nachhaltigen Einfluss nehmen kann». Entscheidend sind die tatsächlich ausgeübte Tätigkeit, die tatsächliche Entscheidungsbefugnis und die tatsächlich wahrgenommene Verantwortung; die formelle Stellung eines Angestellten (z.B. Position im Organigramm usw.) ist nicht erheblich. Dabei ist in erster Linie vom geltenden Arbeitsvertrag auszugehen; die übrigen Umstände der Anstellung sind jedoch mit heranzuziehen. Das zentrale Sachkriterium ist die Entscheidungsbefugnis in wesentlichen Angelegenheiten. Massgeblich ist dafür die tatsächliche Befugnis zu entscheiden, also der sogenannte «letzte Entscheid». Die Möglichkeit, durch Vorschläge oder Anträge auf die Unternehmensführung Einfluss zu nehmen, genügt nicht. Ohne eine tatsächlich leitende Funktion bleibt das ArG damit - unabhängig von der formellen Stellung des Angestellten und von der formellen Bezeichnung seiner Charge - auch auf Leitende Ärzte anwendbar.

b) Oberärzte sind aus dem persönlichen Geltungsbereich des ArG nicht ausgenommen und werden vom Arbeitsgesetz grundsätzlich erfasst. Ausnahme: Das ArG gilt für sie nicht, wenn sie öffentlich-rechtlich an einem öffentlich-rechtlichen Spital ohne eigene Rechtspersönlichkeit angestellt sind.

c) Assistenzärzte waren bisher vom Geltungsbereich des ArG ausgenommen. Auf den 1. Januar 2005 traten jedoch zahlreiche Gesetzes- und Verordnungsänderungen in Kraft. Assistenzärzte wurden aus dem Ausnahmekatalog im ehemaligen Art. 3 lit. e ArG gestrichen und werden neu in Art. 4a der ArGV 1 ausdrücklich als vom ArG erfasst bezeichnet. Seit dem 1. Januar 2005 sind deshalb alle Assistenzärzte, sowohl diejenigen privater als auch öffentlich-rechtlicher Spitäler, ungeachtet deren Rechtsform, dem ArG vollumfänglich unterstellt (Beschlossene Gesetzesänderung vom 22. März 2003, BBl 2002 2746; vgl. BGE 2P.251/2A.407/2001 vom 14. Juni 2002).

Der Übersichtlichkeit halber soll das Ausgeführte anhand einer Tabelle verdeutlicht werden.

\section{Folgen der Anwendbarkeit des ArG}

Wer vom Arbeitsgesetz erfasst wird, erfährt u.a. umfassenden Schutz in den Bereichen Arbeitsund Ruhezeit. Es bestehen tägliche, wöchentliche und jährliche Höchstarbeitszeiten, die jedoch allesamt unter besonderen Voraussetzungen überschritten werden dürfen. Es würde zu weit führen, hier alle Folgen der Anwendbarkeit des ArG aufzuzählen, zumal je nach Betrieb und Arbeitsorganisation verschiedenste Ausnahmen von den gesetzlichen Vorschriften möglich sind. Dem interessierten Leser sei der Blick ins Gesetz ab Art. 9 empfohlen, im Internet findet sich das ArG unter folgendem link: www.admin.ch/ch/ d/sr/c822_11.html. 
Tabelle 1

Übersicht.

\begin{tabular}{|c|c|c|c|c|}
\hline & \multicolumn{4}{|c|}{ Art des Spitals } \\
\hline & Privatspital & $\begin{array}{l}\text { Öffentlich-rechtliches Spital } \\
\text { mit Rechtspersönlichkeit }{ }^{1}\end{array}$ & $\begin{array}{l}\text { Öffentlich-rechtliches Spital } \\
\text { ohne Rechtspersönlichkeit'; } \\
\text { die Mehrzahl der Arbeitnehmer } \\
\text { ist privatrechtlich angestellt }{ }^{2}\end{array}$ & $\begin{array}{l}\text { Öffentlich-rechtliches Spital } \\
\text { ohne Rechtspersönlichkeit }{ }^{\prime} ; \\
\text { die Mehrzahl der Arbeitnehmer } \\
\text { ist öffentlichrechtlich angestellt }{ }^{2}\end{array}$ \\
\hline \multicolumn{5}{|l|}{$\begin{array}{l}\text { Findet das ArG } \\
\text { Anwendung }\end{array}$} \\
\hline auf das Spital? & $\mathrm{Ja}^{3}$ & $\mathrm{Ja}^{3}$ & $\mathrm{Ja}^{3}$ & Nein ${ }^{4}$ \\
\hline \multicolumn{5}{|l|}{$\begin{array}{l}\text { auf Chef- und } \\
\text { leitende Ärzte? }\end{array}$} \\
\hline $\begin{array}{l}\text { - die privatrechtlich } \\
\text { angestellt sind? }\end{array}$ & Nein ${ }^{5}$ & Nein ${ }^{5}$ & Nein ${ }^{5}$ & Nein ${ }^{5}$ \\
\hline $\begin{array}{l}\text { - die öffentlich-rechtlich } \\
\text { angestellt sind? }\end{array}$ & Nein ${ }^{5}$ & Nein ${ }^{5}$ & Nein ${ }^{5}$ & Nein, nie \\
\hline \multicolumn{5}{|l|}{ auf Oberärzte? } \\
\hline $\begin{array}{l}\text { - die privatrechtlich } \\
\text { angestellt sind? }\end{array}$ & Ja & Ja & Ja & Ja \\
\hline $\begin{array}{l}\text { - die öffentlich-rechtlich } \\
\text { angestellt sind? }\end{array}$ & $\mathrm{Ja}$ & $\mathrm{Ja}$ & $\mathrm{Ja}$ & Nein \\
\hline auf Assistenzärzte? & $\mathrm{Ja}$ & $\mathrm{Ja}$ & $\mathrm{Ja}$ & Ja \\
\hline $\begin{array}{l}\text { Ist Art. 3a ArG } \\
\text { (Gesundheitsschutz) } \\
\text { anwendbar? }\end{array}$ & $\mathrm{Ja}$ & Ja & $\mathrm{Ja}$ & Ja \\
\hline & \multicolumn{4}{|c|}{$1 \mathrm{Ob}$ das Spital als solches mit oder ohne Rechtspersönlichkeit ausgestaltet ist, wird in den Statuten geregelt. } \\
\hline & \multicolumn{4}{|c|}{$\begin{array}{l}2 \text { Ob die Arbeitnehmer mehrheitlich öffentlich- oder privatrechtlich angestellt sind, bestimmt sich nur nach den im jeweiligen } \\
\text { Betrieb tatsächlich vorhandenen Arbeitsverhältnissen. Der Kanton ist in der Regelung der Natur des Anstellungsverhältnisses } \\
\text { des Spitalarztes zum öffentlich-rechtlichen Spital frei. }\end{array}$} \\
\hline & \multicolumn{4}{|c|}{$\begin{array}{l}3 \text { Vorbehalt aus Art. } 71 \text { lit. b ArG: Das ArG gilt in diesem Bereich nur subsidiär, das öffentliche Dienstrecht geht inm vor. } \\
\text { Das ArG gilt aber im Bereich von Gesundheitsschutz und Arbeits- und Ruhezeiten als Mindestschutz. }\end{array}$} \\
\hline & \multicolumn{4}{|c|}{$\begin{array}{l}4 \text { Vorbehalt aus Art. } 4 a \text { ArGV 1: Im Rahmen von Arbeitsverhältnissen mit Assistenzärzten ist das ArG auch auf solche Spitäler } \\
\text { anwendbar. In diesem Falle ist der Vorbehalt in Fussnote } 3 \text { zu beachten. }\end{array}$} \\
\hline & \multicolumn{4}{|c|}{5 Falls aber eine tatsächlich leitende Tätigkeit nicht vorliegt: Ja. } \\
\hline
\end{tabular}

Im gleichen Atemzug gilt es festzuhalten, dass der Umsetzungsprozess dieser Neuerungen von allen Beteiligten viel Verständnis und Flexibilität fordern wird. Den Fokus allein auf Fragen der Arbeits- und Ruhezeit zu legen wäre ebenso falsch wie das Verschliessen der Augen vor der neuen gesetzlichen Realität. Vielmehr muss das an den einzelnen Spitälern zweifellos bestehende Optimierungspotential in einem konstruktiven Prozess ausgelotet und umgesetzt werden. Dass dieser Vorgang viel Zeit in Anspruch nehmen wird, versteht sich von selbst. 